\section{OPEN ACCESS}

Edited by:

Xulin Chen,

Jinan University, China

Reviewed by:

Johan Van Weyenbergh,

KU Leuven, Belgium

Ian Christopher Davis,

The Ohio State University,

United States

*Correspondence:

Lu Lu

Ilu@uthsc.edu

Klaus Schughart

klaus.schughart@helmholtz-hzi.de

Robert W. Williams

rwilliams@uthsc.edu

${ }^{\dagger}$ Present address:

Amy C. Sims,

Pacific Northwest National Laboratory in Richland, WA, United States

¥These authors have contributed equally to this work

Specialty section:

This article was submitted to Viral Immunology,

a section of the journal

Frontiers in Immunology

Received: 16 September 2020

Accepted: 19 November 2020

Published: 08 January 2021

Citation:

Xu F, Gao J, Bergmann S, Sims AC,

Ashbrook DG, Baric RS, Cui Y,

Jonsson CB, Li K, Williams RW,

Schughart K and Lu L (2021) Genetic

Dissection of the Regulatory

Mechanisms of Ace2 in the Infected Mouse Lung.

Front. Immunol. 11:607314.

doi: 10.3389/fimmu.2020.607314

\title{
Genetic Dissection of the Regulatory Mechanisms of Ace2 in the Infected Mouse Lung
}

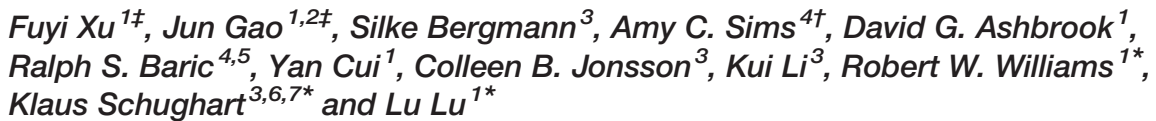

${ }^{1}$ Department of Genetics, Genomics, and Informatics, University of Tennessee Health Science Center, Memphis, TN, United States, ${ }^{2}$ Institute of Animal Husbandry and Veterinary Science, Shanghai Academy of Agricultural Sciences, Shanghai, China, ${ }^{3}$ Department of Microbiology, Immunology, and Biochemistry, University of Tennessee Health Science Center, Memphis, TN, United States, ${ }^{4}$ Department of Epidemiology, University of North Carolina at Chapel Hill, Chapel Hill, NC, United States, ${ }^{5}$ Department of Microbiology and Immunology, University of North Carolina at Chapel Hill, Chapel Hill, NC, United States, 6 Department of Infection Genetics, Helmholtz Centre for Infection Research, Braunschweig, Germany, 7 University of Veterinary Medicine Hannover, Hannover, Germany

Acute lung injury (ALI) is an important cause of morbidity and mortality after viral infections, including influenza A virus H1N1, SARS-CoV, MERS-CoV, and SARS-CoV-2. The angiotensin I converting enzyme 2 (ACE2) is a key host membrane-bound protein that modulates ALI induced by viral infection, pulmonary acid aspiration, and sepsis. However, the contributions of ACE2 sequence variants to individual differences in disease risk and severity after viral infection are not understood. In this study, we quantified H1N1 influenza-infected lung transcriptomes across a family of 41 BXD recombinant inbred strains of mice and both parents-C57BL/6J and DBA/2J. In response to infection Ace2 mRNA levels decreased significantly for both parental strains and the expression levels was associated with disease severity (body weight loss) and viral load (expression levels of viral NA segment) across the BXD family members. Pulmonary RNA-seq for 43 lines was analyzed using weighted gene co-expression network analysis (WGCNA) and Bayesian network approaches. Ace2 not only participated in virusinduced ALI by interacting with TNF, MAPK, and NOTCH signaling pathways, but was also linked with high confidence to gene products that have important functions in the pulmonary epithelium, including Rnf128, Muc5b, and Tmprss2. Comparable sets of transcripts were also highlighted in parallel studies of human SARS-CoV-infected primary human airway epithelial cells. Using conventional mapping methods, we determined that weight loss at two and three days after viral infection maps to chromosome $\mathrm{X}$-the location of Ace2. This finding motivated the hierarchical Bayesian network analysis, which defined molecular endophenotypes of lung infection linked to Ace2 expression and to a key disease outcome. Core members of this Bayesian network include Ace2, Atf4, Csf2, Cxcl2, Lif, Mam/3, Muc5b, Reg3g, Ripk3, and Traf3. Collectively, these findings define a causally-rooted Ace2 modulatory network relevant to host response to viral infection and identify potential therapeutic targets for virus-induced respiratory diseases, including those caused by influenza and coronaviruses. 


\section{INTRODUCTION}

Five major influenza A virus (IAV) pandemics have swept through human populations in the last 130 years-the 1890 H3N8 pandemic, the devastating $1918 \mathrm{H} 1 \mathrm{~N} 1$ pandemic (1), and serious global outbreaks in 1957 (H2N2), 1968 (H3N2), and the 2009 H1N1 swine influenza. The current COVID-19 pandemic is caused by a novel coronavirus, termed severe acute respiratory syndrome (SARS) coronavirus 2 (SARS-CoV-2). This pathogen has spread with unprecedented speed and efficiency from an initial outbreak in November or December 2019.

The primary pathological features of the influenza A outbreaks and both the 2002 SARS and current COVID-19 outbreaks are viral pneumonia and acute lung injury (ALI) (2). The well documented symptoms of influenza A (H1N1) and COVID-19 have substantial overlap. For both viral pathogens, infected humans can have symptoms ranging from mild coughing, congestion, and fever, to severe and lethal pulmonary edema and disseminating diseases of other organs. Individual host genetic differences almost certainly play an important role in the development and severity of the illness and its time course (3-7).

The human angiotensin I converting enzyme 2 (ACE2) is a cell membrane carboxypeptidase and negative modulator of the renin angiotensin system (RAS). This protein also has a key role in ciliary function in epithelial cells and is the principal receptor bound by spike proteins of SARS-CoV $(8,9)$ and SARS-CoV-2 (10). ACE2 is highly expressed in nasal cavity epithelial cells, especially on apical cilia of small airway cells and in alveolar epithelial type II cells (AT2) $(11,12)$.

Paradoxically, while serving as a key node in cellular entry of SARS-CoV and SARS-CoV-2 virions, ACE2 also protects against lung disease in animal models (13). For example, treatment with recombinant human ACE2 protects against acute lung failure induced by acid aspiration or sepsis in Ace 2 knockout mice as well as in wild-type controls (14). Of note, Ace2 expression in mouse lungs is downregulated following SARS-CoV infection. The spike protein alone induces ACE2 downregulation in cell culture and enhances the severity of ALI induced by acid aspiration in wildtype mice (8). In contrast, viral spike protein is unable to affect the severity of lung failure in Ace2 knockout animals, indicating the effect of spike protein on acid-induced ALI is ACE2 specific (8). Interestingly, expression of ACE2 is also significantly downregulated by IAVs in infected cell cultures, although in this case, ACE2 is dispensable for viral entry and replication (15). In mice, Ace2 deficiency is associated with aggravated ALI induced by IAV H7N9 or respiratory syncytial virus $(16,17)$. The precise mechanism underlying these findings is unclear, as is how alterations in ACE2 expression modulate the severity of pulmonary disease inflicted by coronaviruses and other IAV subtypes.

Expression of ACE2 in human lung has an impressive level of variation among healthy humans. In the GTEx RNA-seq transcriptome survey (18), the maximum range of expression in the lung is about 22-fold, the interquartile range is 1.8 -fold, and the coefficient of variation (standard deviation/mean) is high-nearly 0.6 (genenetwork.org/show_trait?trait_id=ENSG00000130234 \&dataset=GTEXv8_Lung_tpm_0220). Given this finding, it is possible, even likely, that there are marked genetic differences in host susceptibility that are a function of numbers or ratios of ACE2positive AT2 cells and epithelial cells in lung (11) or the level expression by these cells of ACE2. However, we emphasize that causal linkage between genetic variation in ACE2 and to susceptibility or progress of infection is still poorly defined in humans, or even in mouse models.

Susceptibility to IAV, such as H1N1 and H7N7 influenza infections, varies greatly among strains of mice (19), and especially between the two key inbred strains we use hereC57BL/6J (B6), the reference strain for most genetic and molecular studies-and DBA/2J (D2), the oldest inbred strain of mice (20). B6 is relatively resistant to IAV and often survives infection. In contrast, D2 loses body weight rapidly, and at the same inoculum dose as given to B6 dies within 5-7 days. Our laboratory has generated and maintains approximately 120 BXD recombinant inbred (RI) strains derived from crosses between B6 and D2 parents. Collectively, this family of RI strains segregates for $\sim 6$ million variants with minor allele frequencies above 0.3 (21), making it an ideal reference population to model genetic variation in susceptibility to IAV infections (22). Indeed, in previous genetic mapping using the BXD family we have defined loci and even cloned gene variants that control the host response to IAV (H1N1) infection (23) and to other infectious diseases (24-27). Here, we have analyzed the lung transcriptome of 41 members of the family and both parental strains after infection with IAV (H1N1). We define patterns of covariation of RNA-seq data with induced variation in Ace 2 expression, as well as with outcome measures and with key molecular networks and endophenotypes likely to contribute to host defense and pathologies of $\mathrm{H} 1 \mathrm{~N} 1$ and possibly to SARS$\mathrm{CoV}$-induced ALI.

\section{MATERIALS AND METHODS}

\section{Mice}

We used females from 41 BXD RI strains and both parental strains-B6 and D2. Mice were between 8 and 12 weeks of age when infected. They were housed and maintained on a 12:12 light/dark cycle, with ad libitum access to food and water.

\section{Virus}

Original stocks of mouse-adapted A/Puerto Rico/8/34 (H1N1, PR8M) virus were obtained from Stefan Ludwig, University of Münster (28). Virus stocks were propagated in the chorioallantoic cavity of 10-day-old pathogen-free embryonated chicken eggs for $48 \mathrm{~h}$ at $37^{\circ} \mathrm{C}$ as described previously (29). Viral titer was determined using a focus-forming unit (FFU) assay as described previously (29).

\section{Infection of Mice}

Animals were anesthetized by intraperitoneal injection of ketamine/xylazine $(10 \%(\mathrm{v} / \mathrm{v})$ of $100 \mathrm{mg} / \mathrm{ml}$ ketamine and $5 \%$ (v/v) of $20 \mathrm{mg} / \mathrm{ml}$ xylazine in $0.9 \%(\mathrm{w} / \mathrm{v}) \mathrm{NaCl}$ with a dose adjusted to body weight $(200 \mu \mathrm{l} / 20 \mathrm{~g}$ body weight). Infection was performed by intranasal application of virus solution in $20 \mu \mathrm{l}$ sterile phosphate-buffered saline (PBS), with a PR8M dosage of 
$2 \times 10^{3}$ FFU. Mice were bred and infected at the animal facilities at UTHSC.

\section{RNA Isolation and Sequencing}

Mice were sacrificed 3 days post-infection (dpi) and both lungs were extracted and transferred immediately to RNAlater (Qiagen), stored at $4^{\circ} \mathrm{C}$ for one day, and then stored at $-20^{\circ} \mathrm{C}$. RNA was isolated using Qiagen Midi kit (30). RNA quality was evaluated on a 2100 Bioanalyzer (Agilent). Five-hundred nanograms of total RNA was used to prepare libraries for sequencing using the Lexogen SENSE RNA-seq library kit for Ion Torrent. Libraries were amplified for 11 cycles as the final step of library preparation. Before sequencing, 1- $\mu$ l aliquots were pooled and sequenced on an Ion Torrent PGM 314 chip. Barcoded data from the PGM was used to balance the final pool before sequencing. Library pools were sized to $\sim 260$ bp on a Pippin Prep instrument using 2\% Pippin agarose gel. The sized libraries were evaluated on an Agilent High Sensitivity chip, quantified using real-time PCR, and used to prepare beads using a One-Touch 2 device. Beads were sequenced on an Ion Torrent Proton P1 chip. On average, 67 million reads were obtained per strain.

\section{Read Mapping and Gene Expression Quantification}

RNA-seq reads were quality-trimmed using Trim Galore (31) and mapped to the mm10 reference genome or to the IAV PR8M genome using STAR (32). Counts were summarized at the gene level using the R-package Rsubread (33), normalized and log transformed using the R-package DESeq2 (34), and batchcorrected using the ComBat function of the R-package sva (35, 36). For annotations of genes, ENTREZID from Rsubread were matched to RefSeq annotations using R-package biomart (37).

\section{Heritability Estimates for Ace2}

Heritability of Ace2 gene expression was estimated with GEMMA (38) using the following formula. This approach estimates the proportion of variance that can be explained (PVE) by genotypes covering nearly the whole genome:

$$
\operatorname{PVE}(\beta, \mathrm{u}, \tau):=\frac{\mathrm{V}(\mathrm{X} \beta+\mathrm{u})}{\mathrm{V}(\mathrm{X} \beta+\mathrm{u})+\tau^{-1}}
$$

where "X $\beta$ " is the sparse effects, " $u$ " is the random effects, and " $\tau^{-1}$ " is the variance of the residual errors. GEMMA uses a Markov chain Monte Carlo method to estimate $\beta$, and $\mathrm{u}$. We used the BXD genotype file generated on January 2017 (www. genenetwork.org/webqtl/main.py?FormID=sharinginfo\&GN_ AccessionId=600) and Ace 2 gene expression values across the 41 BXD family as input.

\section{Correlation Analysis}

The correlation analysis in this study, including gene-gene and gene-phenotype rely on Pearson correlation coefficients (39). We reviewed data to ensure that outliers and distributional anomalies did not compromise downstream analyses.

\section{Weighted Gene Co-Expression Network Analysis}

Weighted Gene Co-Expression Network Analysis (WGCNA) is a method that defines modules of co-expression, and that is often used to explore the associations between variation in mRNA levels and phenotypes of interest. It can be used to define hub genes and clusters in molecular and genetic networks. In this study, gene co-expression networks were constructed using the WGCNA package (40) following standard methods (https:// horvath.genetics.ucla.edu/html/CoexpressionNetwork/ Rpackages/WGCNA/Tutorials/). Modules were generated using the subset of $5500 \mathrm{H} 1 \mathrm{~N} 1$-infected lung mRNAs that covaried well with Ace 2 expression (Pearson $r$ ) at a nominal $p<0.05$. We used the standard WGCNA beta weighting parameter of 8 to achieve close to a scale-free distribution.

\section{Gene Set Enrichment Analysis}

To understand the biological function and clusters detected as Ace 2 covariates and WGCNA modules, we compared set membership to Kyoto Encyclopedia of Genes and Genomes (KEGG) pathways and to the mammalian phenotype ontology (MPO) using WebGestalt (http://www.webgestalt.org) (41). This analysis uses a hypergeometric statistic test to generate adjusted $p$ values and enrichment ratios. In this analysis, we used only protein coding genes as the reference background and a threshold minimum of five genes/transcripts per category.

\section{Quantitative Trait Loci Mapping}

Quantitative Trait Loci (QTLs) define chromosomal regions that contribute to genetic variation in levels of gene expression, endophenotypes, and higher order outcome variables such as body weight loss and survival after infection. QTL mapping exploited methods in our GeneNetwork (www.genenetwork.org) $(42,43)$. Linkage statistics were computed initially using HaleyKnott equations (44), and genome-wide significance levels were estimated from 2,000 permutations of trait values. We used the new BXD genotype file of January 2017 (22). Those traits associated with significant and suggestive QTLs or expression QTLs (eQTLs) using the Haley and Knott regression were validated using GEMMA (38). GEMMA uses a linear mixed model mapping algorithm that accounts for complex kinship among strain genometypes.

\section{Genetic Variations Between B6 and D2}

Sequence differences segregating among BXDs are taken from our previous work (45). Here, we focus exclusively on proteincoding variants-nonsense, missense, and frameshift mutations.

\section{Bayesian Network Construction}

The Bayesian Network Webserver (BNW, compbio.uthsc.edu/ BNW) (46-48) was used to create and evaluate plausible causal models among genes, transcripts, and outcome measures. The 100 highest scoring networks were used for model averaging, and those directed edges-unidirectional connections between nodes-with posterior probabilities greater than 0.8 were retained in the final model. We constrained each node to have 
at most four direct parents. We assigned nodes into one of three tiers of the Bayesian network: 1) SNP variant within selected QTL, 2) mRNA expression traits in a molecular endophenotype tier, and 3) the disease outcome measures-Body Weight Loss in the third tier. We allowed causal connections going from the first to second, and from second to the third tiers.

\section{RNA-Seq Time-Course Data for H1N1 Infection of the Parental Strains}

Lung RNA-seq data was generated previously in our laboratory (20). In brief, the two parental strains, B6 and D2, were infected with the $\mathrm{H} 1 \mathrm{~N} 1$ virus at $10-12$ weeks of age as described above. RNA was isolated from lungs of B6 at 1, 3, 5, 8, and $14 \mathrm{dpi}$ and from lungs of D2 at 1, 3, and 5 dpi. Three RNA samples per strain and per time point were used for library preparation and sequencing. Raw counts were extracted, normalized, and $\log 2$ transformed with DEseq2 (34) after adding 1 to all raw counts. In this study, we extracted the Ace 2 expression levels from this dataset. We also performed differential expression analysis of mice at $3 \mathrm{dpi}$ and their mock treated controls using DESeq2 (34) using strain as a covariate. The resulting $p$ values were adjusted using the Benjamini and Hochberg's approach (49) and are referred throughout this paper as adj. $p$ values. We reported transcripts with adj. $p<0.05$, and fold change $(\mathrm{FC})>1.3$ as differentially expressed genes (DEGs). This data set was also used for evaluating the gene expression patterns post-infection.

\section{Transcriptome Data for SARS-CoV Infected Primary Human Airway Epithelial (HAE) Cells}

The experimental details for this study are described in ref. (50). In brief, human tracheobronchial epithelial cells were resected from airway specimens of patients undergoing surgery. Primary cells were expanded to generate passage- 1 cells, and passage- 2 cells were plated at a density of $2.5 \times 10^{5}$ cells per well. Human airway epithelium (HAE) cultures were generated by provision of an air-liquid interface for 6 to 8 weeks to form well-differentiated, polarized cultures. Wild-type infectious clone-derived SARS-CoV (icSARS$\mathrm{CoV}$ ) and ic-SARS-CoV ORF6 were derived from infectious clone constructs as described (50). Bat-SARS-CoV is comprised of the HKU3 backbone and SARS-CoV receptor binding domain and was derived from an infectious clone as described in ref. (51). Triplicate cultures were infected with a multiplicity of infection 3 (MOI 3) icSARS-CoV, and icSARS-CoV ORF6 on the apical surface. Total RNA was isolated from infected cells, and equivalent amounts of RNA from three biological replicates from each condition were pooled. Transcriptome profiling was performed using Agilent $4 \mathrm{x} 44 \mathrm{~K}$ whole human gene expression arrays. Raw data were downloaded from GEO (GSE47963_series_matrix.txt.gz). Expression values were normalized and sample descriptions quality controlled. A strong batch effect with respect to the donor from which $\mathrm{HAE}$ were derived was corrected using the $\mathrm{R}$ package sva $(35,36)$. Rows with no gene description were deleted. DEGs were determined using the R package Limma (52).

\section{Data Availability}

The RNA-seq data from infected BXD strains has been deposited at GeneNetwork.org under the accession ID GN807. The summarized RNA-seq data as entered in GN can be accessed and downloaded at www.genenetwork.org/webqtl/main.py? FormID $=$ sharinginfo\&GN_AccessionId=807\&InfoPageName $=$ HZI_LungBXD_RNA-Seq_1116.

RNA-seq data for infected B6 and D2 parents are available at NCBI GEO under the accession number GSE66040. Array data from infected primary HAE cells are available at GEO under accession number GSE47963.

\section{RESULTS}

\section{Ace2 Expression Level Decreased in Both B6 and D2 After H1N1 Infection}

We have previously generated H1N1-inflected lung RNA-seq for B6 and D2 parental strains at 1, 3, 5, 8, and 14 dpi. The B6 maternal parent of the BXDs is resistant to H1N1 infection, whereas the D2 paternal parent is highly susceptible to low virulent PR8 (H1N1) virus. Here, we have exploited these parental time-series data to define the Ace 2 mRNA post-infection dynamic response. Overall, expression of Ace 2 in lungs dropped progressively in both strains after infection (Figure 1). In B6, Ace 2 transcript reached its nadir at $8 \mathrm{dpi}(7.20 \pm 0.15 \mathrm{SD} \log 2$ units) and stayed low at $14 \mathrm{dpi}(7.23 \pm$ 0.12 ). Expression relative to 1 dpi was significantly lower at the following time points: $3 \mathrm{dpi}(p=0.026, \log 2 \mathrm{FC}=-0.25), 5 \mathrm{dpi}(p=$ $0.008, \log 2 \mathrm{FC}=-0.49), 8 \mathrm{dpi}(p=0.003, \log 2 \mathrm{FC}=-0.73)$, and 14 dpi $(p=0.002, \log 2 \mathrm{FC}=-0.70)$. In comparison, the same infection titer was lethal for D2 which died at $5 \mathrm{dpi}$, and this was matched with lower expression of Ace2 (7.10 \pm 0.25$)$ at 1 dpi. Statistically,

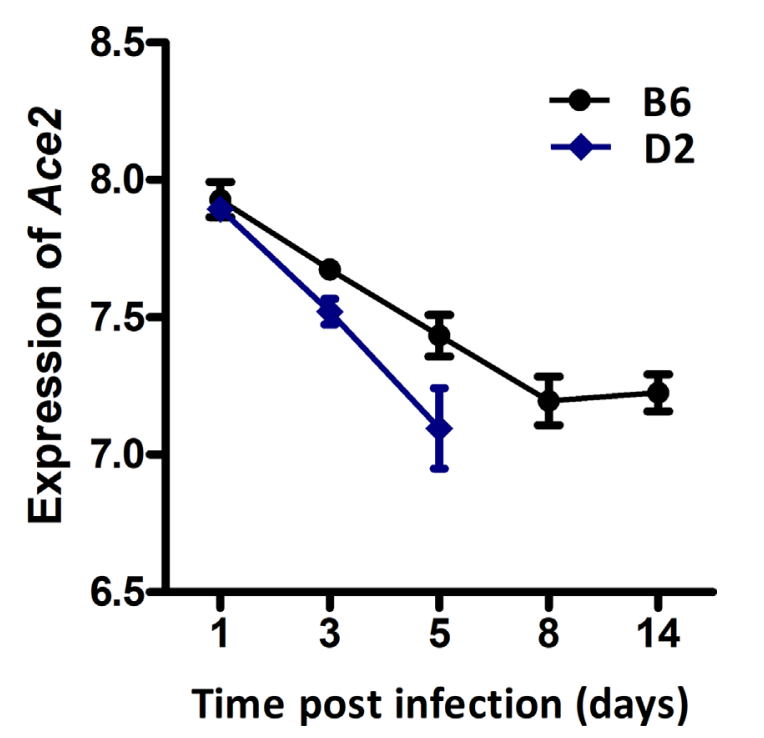

FIGURE 1 | Line charts of the Ace2 expression level in strains C57BL/6J (B6) and DBA/2J (D2) after H1N1 infection. Lung transcriptomes were quantified with RNA-seq for B6 and D2 strains at 1, 3, 5, 8, and 14 dpi of H1N1 infection. Three female mice (8-12 weeks of age) per strain were used per time point. The $x$-axis shows the time point (days) post the infection. The $y$-axis shows the expression level of Ace2 on a log2 transformed scale. 
Ace 2 expression $3 \mathrm{dpi}(p=0.003, \log 2 \mathrm{FC}=-0.37)$ and $5 \mathrm{dpi}(p=$ $0.006, \log 2 \mathrm{FC}=-0.80$ ) were significant reduced compared to $1 \mathrm{dpi}$. Although the differences in Ace 2 expression between B6 and D2 at 3 dpi and 5 dpi were not statistically significant, D2 mice had a consistently more rapid decline in expression compared to B6.

\section{Ace2 Expression Exhibited Variability Across BXD Strains at $3 \mathrm{dpi}$}

All infected D2 mice died at 5 dpi. We therefore generated RNA-seq data at 3 dpi for infected BXD strains. Changes in Ace 2 transcript level after infection varies considerably across the BXD family with a 1.5-fold range (Figure 2). Mean expression of Ace2 across all 43 strains is $8.02 \pm 0.15 \mathrm{SD}$. BXD22 has the lowest expression of 7.78 and BXD77 has the highest expression of 8.36. The estimated heritability of Ace 2 expression variation is $\sim 0.18$, suggesting that expression of $A c e 2$ is partly impacted by genetic factors.

\section{Ace2 Is Associated With Disease Severity After Infection}

In order to investigate whether there is an association of Ace2 expression with severity of disease and/or viral replication as infection progresses, we computed correlations of Ace 2 expression against body weight loss, mean time to death, and viral gene expression levels (as a surrogate for viral load). Ace2 expression was significantly correlated with both body weight loss $(r=0.575$, $p=0.008$, Figure 3A) and median body weight loss $(r=0.468, p=$ 0.028 , Figure 3B) at $3 \mathrm{dpi}$. Further, Ace 2 was positively and significantly correlated with the mean time to death $(r=0.458$, $p=0.032$, Figure 3C). In contrast, Ace 2 had a negative significant correlation with virus NA gene expression levels $(r=-0.381, p=$ 0.029, Figure 3D).

\section{Ace2-Correlated Transcripts Are Significantly Involved in Immune System- Related Networks}

To gain insight into the pulmonary networks and biological functions that $A c e 2$ is involved in after viral infection, we computed Pearson correlation coefficients against the entire lung transcriptome. This analysis returned 5,500 transcripts nominally correlated with Ace2 ( $p<0.05$, Supplemental Data 1).
A total of 5,118 transcripts with Entrez gene IDs were submitted to WebGestalt (http://www.webgestalt.org/) for gene ontology analyses. The analysis highlighted strong enrichment in TNF signaling, MAPK signaling, Notch signaling pathways, HTLV-I infection, and VEGF signaling pathway (Figure 4A). Furthermore, we also found that these genes were enriched for categories related to chemokine secretion, lung morphology, and respiratory system morphology (Figure 4B).

Forty-eight genes are within the TNF signaling pathway (Supplemental Data 1), of which most have transcripts that covary negatively $(n=35)$ with Ace $2(p<0.05)$, including tumor necrosis factor (Tnf, $r=-0.414, p=0.005$ ). Ninty-two genes are part of the extended MAPK signaling network (Supplemental Data 1). Of these $T g f b 1(r=-0.459, p=0.002)$ and 59 other transcripts covary negatively with Ace2. Finally, 25 genes are part of Notch signaling pathways (Supplemental Data 1), and again the majority of transcripts $(n=21)$ covary negatively with Ace2.

\section{Co-Expression Network Analysis Narrowed Down Ace2-Correlated Transcripts}

To identify genes that interact more directly with Ace2, we constructed co-expression modules using WGCNA with Ace2 correlated transcripts. The top most variant 5,500 transcripts were parsed into 10 co-expression modules using a softthresholding power of 8 (Figures 5A-C). Modules have a wide range of sizes, with a brown module containing 1,673 transcripts and lightcyan containing only 68 transcripts. Ace 2 was located in the purple module with 461 other genes/transcripts (Figure 5C). Genes in this module are significantly involved in cilium function (Figure 5D)

In subsequent analyses we focused on transcripts within the purple module (Supplemental Data 2). We carried out differential expression analysis between cases and mockinfected controls and defined 281 transcripts $(61 \%$ of the module) with differential expression (adj. $p<0.05$ and FC $>$ 1.3). eQTL mapping of the H1N1-infected whole lung transcriptome defined 23 cis-modulate transcripts and 106 trans-modulated transcripts, of which 69 are likely to harbor coding sequence variants. A subset of 42 of these genes has been

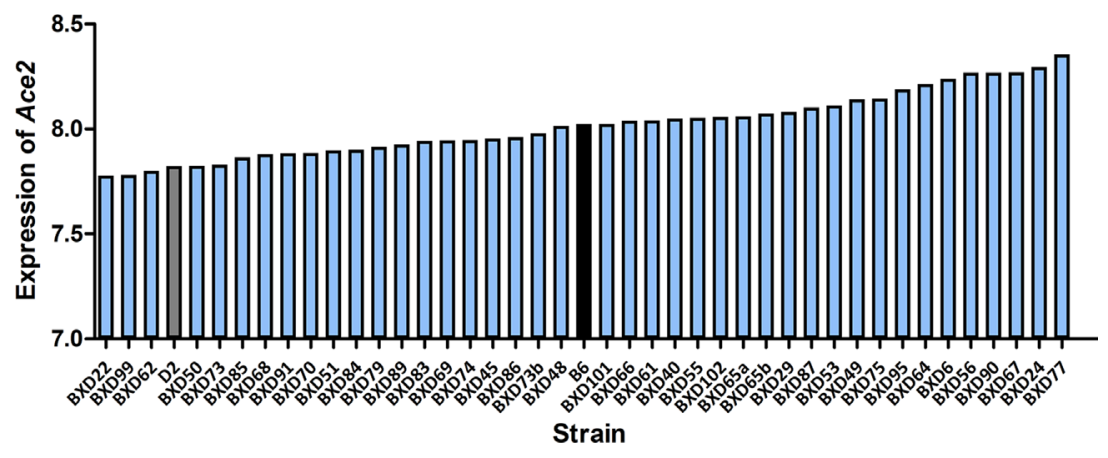

FIGURE 2 | Expression of Ace2 in the BXD family. Bar plots of the pulmonary Ace2 expression levels across the BXD family at 3 dpi. The X-axis shows BXDs strains and the two parental strains. The y-axis shows the normalized log2 expression levels of Ace2. 

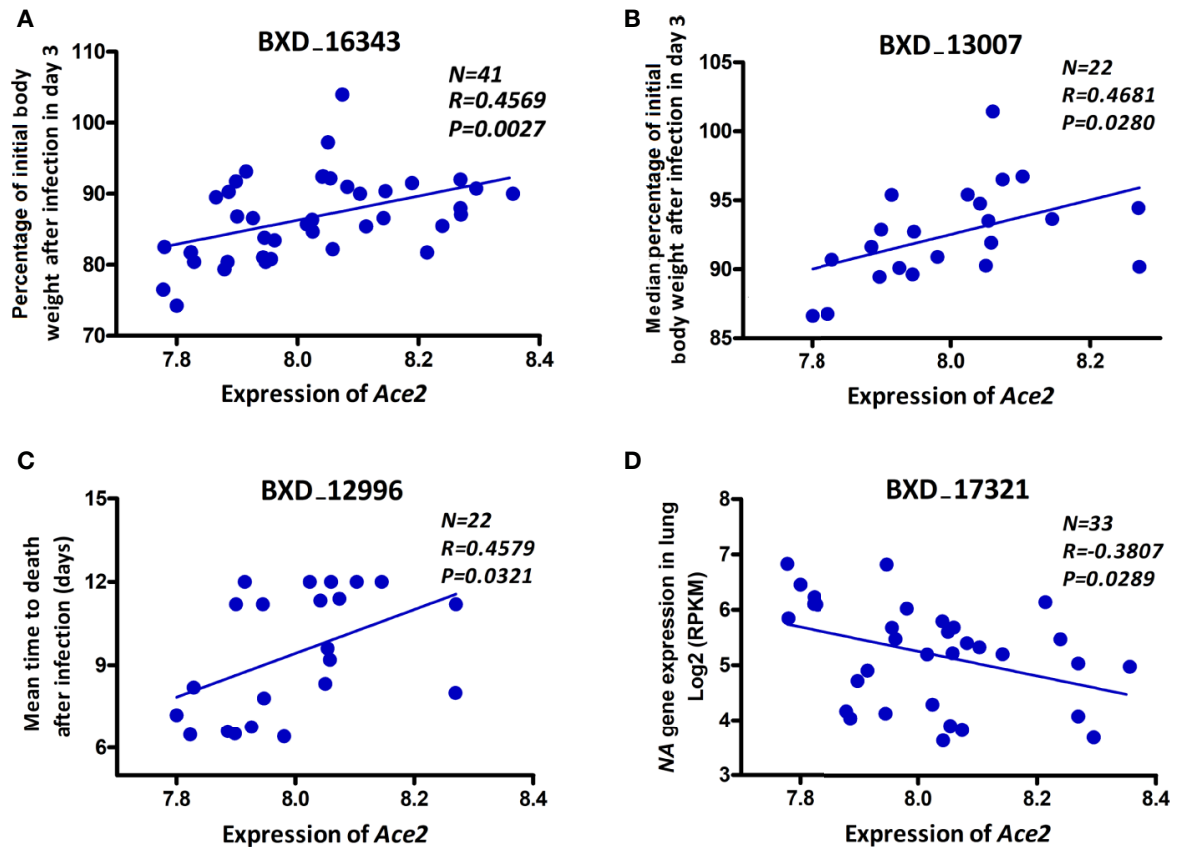

FIGURE 3 | Ace2 is associated with disease severity after infection. Scatter plots of the correlations of Ace2 expression with body weight loss (A), median body weight loss (B) at 3 dpi, mean time to death (C), and viral neuraminidase (NA) gene expression levels (D). Pearson correlation coefficient was used to determine the relationship. Gene expression levels represent normalized log2 values. GeneNetwork BXD phenotype identifiers (e.g., ID "BXD_16343") are at the top of each plot. Number of strains $(\mathrm{N})$, Pearson correlation R-value $(\mathrm{R})$, and Pearson correlation P-value $(\mathrm{P})$ are indicated. BXD trait 16343 and BXD trait 17321: one mouse per strain, BXD trait 13007 and BXD trait 12996: five mice per strain.
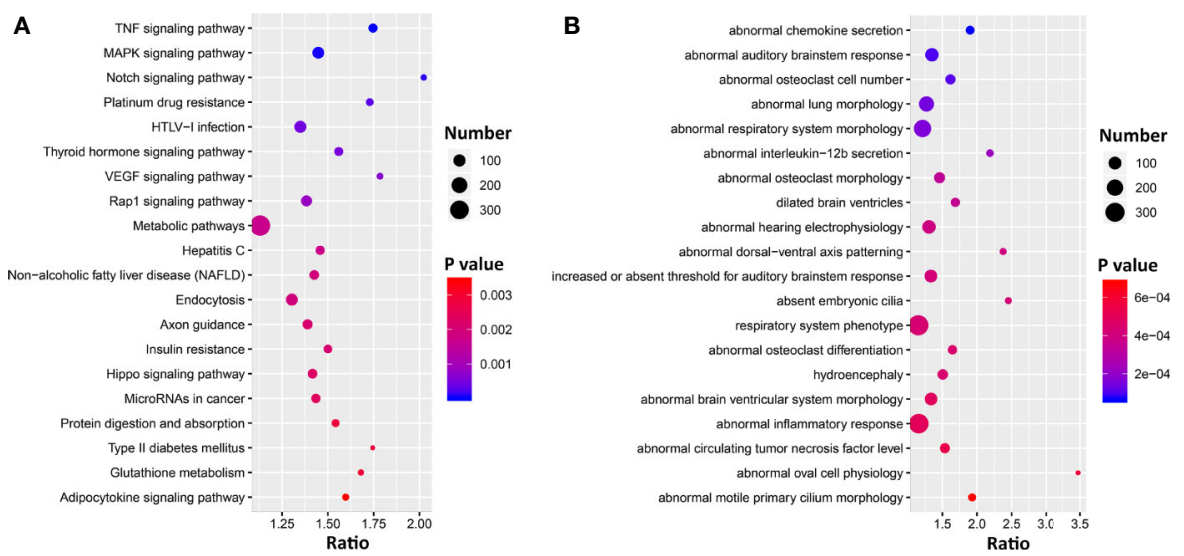

FIGURE 4 | Bubble charts of the top 20 KEGG pathways (A) and 20 Mammalian Phenotype Ontologies (B) enriched for Ace2 significantly correlated genes. Gene over-representation analysis for KEGG pathway and Mammalian Phenotype Ontology of the Ace2 correlated transcripts $(p<0.05)$ were performed with WebGestalt (www.webgestalt.org). The $x$-axis represents the enrich ratio and the $y$-axis represents enriched pathways/terms. The size of the dots denotes the number of genes and the color denotes the $p$-value.

implicated in cilium function. By comparing the above four categories (Figure 6), 31 cilium genes were differentially expressed (Table 1). Of these, Sox11, Tff2, Rnf128, and Muc5b are either cis- or trans-controlled, whereas Sox11, Tmprss2, Ulk4, Cckar, Wdpcp, Ift140, and Ccdc40 contain functional variants (Table 1).
The Differentially Expressed Cilium Function Related Genes in the Purple Module Were Replicated in SARS-CoVInfected HAE Cells

In order to evaluate whether cilium related genes (Table 1) are also modulated in humans, especially in the context of SARS- 

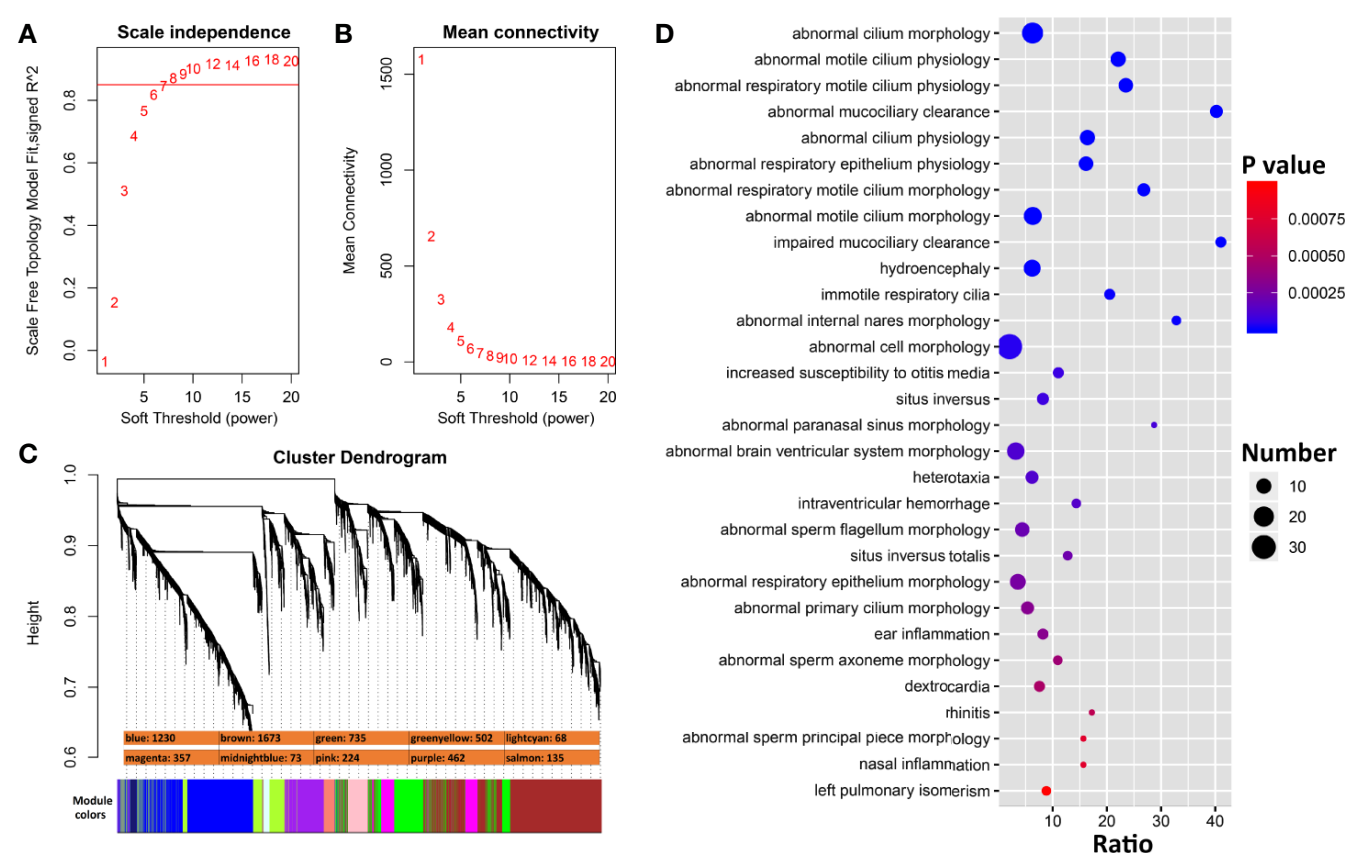

FIGURE 5 | WGCNA modules associated with Ace2 expression in $\mathrm{H} 1 \mathrm{~N} 1$ infected lung. (A) The soft thresholding index $\mathrm{R}^{2}$ ( $y$-axis) as a function of different powers $\beta$ ( $\mathrm{X}$-axis) indicating the threshold of 8 chosen. (B) Mean connectivity (degree, $y$-axis) as a function of the soft-thresholding power ( $\mathrm{X}$-axis). (C) 10 co-expression modules identified from Ace2 correlated genes. Modules are color coded as shown. The number of genes in each module is also indicated. (D) Bubble charts of top 30 enriched Mammalian Phenotype Ontologies of the purple module. Analysis by WebGestalt.

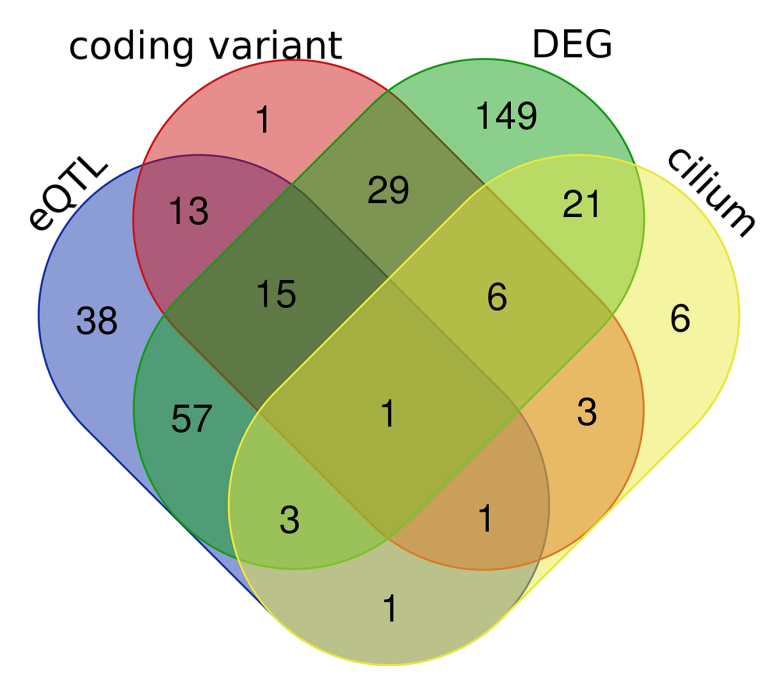

FIGURE 6 | Venn diagrams of the purple module genes. A total of 462 genes were included in the purple module. Four criteria were applied to explore the module genes, including coding sequence variants between parental strain B6 and D2 (coding variant), eQTL (LRS > 15) regulation in H1N1 affected lung tissue (eQTL), cilium functional relevance (cilium), and differential expression (adj. $p<0.05$ and FC $>1.3$ ) between infected and normal controls in parental strains (DEG). The Venn diagram shows the number of overlapping genes among the four categories.
CoV infection, we analyzed our transcriptome data set for HAE cells infected with SARS-CoV (icSARS-CoV), ic-SARS-CoV ORF6 and BAT-SARS_CoV (50). Very few of these transcripts were significantly affected after infection with BAT-SARS (Figure 7A). However, most of them were differentially expressed in icSARS-CoV and ic-SARS-CoV ORF6-infected HAE cells when compared to mock control, with 21 transcripts modulated at $96 \mathrm{~h}$ after infection (Figure 7A). More importantly, those overlapping transcripts, except Ace2, exhibited the same up- or down-expression patterns in both HAE cells and our mouse model (Table 1 and Figure 7B). ACE2 was up-regulated in HAE cultures whereas in whole mouse lungs it was down-regulated. This is an important observation because HAE cultures consist almost exclusively of airway epithelial cells, with few if any interstitial cells, endothelial cells, resident and infiltrating immune cells. This indicates that ACE2 networks detected in whole lungs are much more complex than those detected in isolated epithelial cells. It may be the case that ACE2 is downregulated in all cells in lung after infection, but alternatively, ACE2 may be up-regulated only in infected cells (50, 53). In the lungs, infected cells represent a minority of all cells. Since our lung transcriptome quantifies steady-state expression changes of all cell types, it is conceivable that we do not detect up-regulation in a smaller population of infected cells. Besides, two recent studies (54, 55) show alternative splicing of ACE2 in humans, up-regulated by both viruses and IFNs. Therefore, it may be important to quantify different Ace2 isoforms' expression in infected mouse lungs by 
TABLE 1 | Lists of prioritized genes in the purple module that were differentially expressed (adj. $p<0.05$ and FC > 1.3) and implicated in cilium biological functions.

\begin{tabular}{|c|c|c|c|c|c|c|}
\hline Gene ID & Symbol & Description & Log2 FC & Adj.p & eQTL & Functional Variant \\
\hline 20666 & Sox11 & SRY-box 11 & -1.19 & $1.95 \mathrm{E}-12$ & cis & Nonsynonymous \\
\hline 21785 & Tff2 & trefoil factor 2 (spasmolytic protein 1) & -1.01 & 5.38E-04 & trans & - \\
\hline 66889 & Rnf128 & ring finger protein 128 & -0.77 & 2.81E-05 & trans & - \\
\hline 74180 & Muc5b & mucin 5 , subtype $B$, tracheobronchial & 0.54 & 1.13E-02 & trans & - \\
\hline 50528 & Tmprss2 & transmembrane protease, serine 2 & -1.06 & $8.58 \mathrm{E}-10$ & - & Nonsynonymous \\
\hline 209012 & Ulk4 & unc-51-like kinase 4 (C. elegans) & -0.87 & $3.43 \mathrm{E}-07$ & - & Nonsynonymous \\
\hline 12425 & Cckar & cholecystokinin A receptor & -0.73 & 1.91E-05 & - & Nonsynonymous \\
\hline 216560 & Wdpcp & WD repeat containing planar cell polarity effector & -0.69 & 2.36E-06 & - & Nonsynonymous \\
\hline 106633 & Ift140 & intraflagellar transport 140 homolog (Chlamydomonas) & -0.68 & $3.50 \mathrm{E}-07$ & - & Nonsynonymous \\
\hline 207607 & Ccdc40 & coiled-coil domain containing 40 & -0.66 & 1.06E-03 & - & Nonsynonymous \\
\hline 320752 & Dpy19/2 & dpy-19-like 2 (C. elegans) & -1.81 & 2.13E-03 & - & - \\
\hline 51938 & Ccdc39 & coiled-coil domain containing 39 & -1.30 & $1.15 \mathrm{E}-13$ & - & - \\
\hline 244653 & Hydin & hydrocephalus inducing & -1.30 & $8.51 E-16$ & - & - \\
\hline 74362 & Spag17 & sperm associated antigen 17 & -1.29 & $9.27 \mathrm{E}-13$ & - & - \\
\hline 73873 & Fam161a & family with sequence similarity 161 , member $A$ & -1.28 & 1.70E-09 & - & - \\
\hline 320662 & Casc1 & cancer susceptibility candidate 1 & -1.27 & 2.44E-08 & - & - \\
\hline 66722 & Spag16 & sperm associated antigen 16 & -1.27 & $1.61 \mathrm{E}-10$ & - & - \\
\hline 74934 & Armc4 & armadillo repeat containing 4 & -1.21 & 8.50E-09 & - & - \\
\hline 18703 & Pigr & polymeric immunoglobulin receptor & 1.18 & 1.10E-09 & - & - \\
\hline 69707 & $\operatorname{lqcg}$ & IQ motif containing $G$ & -1.18 & 2.97E-20 & - & - \\
\hline 78801 & $A k 7$ & adenylate kinase 7 & -1.17 & 4.75E-09 & - & - \\
\hline 27078 & B9d1 & B9 protein domain 1 & 1.09 & 1.00E-02 & - & - \\
\hline 68922 & Dnaic1 & dynein, axonemal, intermediate chain 1 & -1.07 & 3.67E-08 & - & - \\
\hline 226265 & Eno4 & enolase 4 & -0.98 & $1.49 \mathrm{E}-09$ & - & - \\
\hline 71877 & Efhc1 & EF-hand domain (C-terminal) containing 1 & -0.92 & 3.77E-11 & - & - \\
\hline 75533 & Nme5 & expressed in non-metastatic cells 5 & -0.82 & $5.92 \mathrm{E}-05$ & - & - \\
\hline 75050 & Kif27 & kinesin family member 27 & -0.71 & 3.05E-03 & - & - \\
\hline 70008 & Ace2 & angiotensin I converting enzyme (peptidyl-dipeptidase A) 2 & -0.61 & $9.88 \mathrm{E}-05$ & - & - \\
\hline 20878 & Aurka & serine/threonine kinase 6 & 0.60 & 2.36E-02 & - & - \\
\hline 22062 & $\operatorname{Trp} 73$ & transformation related protein 73 & -0.60 & $1.81 \mathrm{E}-03$ & - & - \\
\hline 252973 & Grhl2 & grainyhead-like 2 (Drosophila) & -0.44 & 5.36E-04 & - & - \\
\hline
\end{tabular}

immunohistochemistry, by single-cell methods, or by in situ hybridization to resolve specificity and kinetics of responses.

\section{Bayesian Network Analysis of Causal Pathways Underlying Post-Infection Weight Loss}

Body weight loss is an important indicator of post-infection severity. We performed QTL mapping for body weight changes after infection. This allowed us to identify locus on Chr X at 164 $\mathrm{Mb}$ that clearly modulate body weight loss at 2 and $3 \mathrm{dpi}$ (Figures 8A, B), respectively. This locus is precisely aligned to the chromosomal position of the Ace 2 gene (Chr X at $164.2 \mathrm{Mb}$ ), which supports a central hypothesis of this study-that variants in or near Ace2 influence influenza disease severity.

In order to investigate how this locus modulates influenza disease severity, hereafter referred to as body weight loss, we created a Bayesian network model (Figure 8D) comprising genotypes at this QTL location (Chr X: rs31223993) plus its candidate gene Ace 2 as a genetic driver, and body weight loss as the key outcome response. We tested causal networks that included nine transcripts as putative disease mediators. All nine are highly expressed in the lung, have a significant correlation with Ace 2 and body weight loss at 2 and 3 dpi $(p<$ 0.05 ), and have a significant expression change (FC $>1.3$ and adj. $p<0.05)$ after infection at multiple time points between the infected and the mock controls (Figure 8C). Moreover, all of these transcripts and their cognate genes have been implicated in functions related to the infection related pathways (TNF signaling pathway, IL-17 signaling pathway, Th1 and Th2 cell differentiation).

The Bayesian network model (Figure 8D) linked the Chr X QTL for body weight loss outcome at 2 and 3 dpi with multiple gene mediators, including Csf2, Cxcl2, Maml3, Muc5b, Ripk3, andTraf3. It is worth noting that any pathway leading from the major Chr X QTL to body weight loss must pass through Atf4, Lif, or Reg3g. For instance, one causal path for body weight loss begins at the Chr X QTL genotype (rs31223993), continues to the expression of Ace2 and Lif, and finally impacts phenotype outcomes.

\section{DISCUSSION}

Gene variants significantly influence the susceptibility of humans to influenza virus infections (56). This is just as true in mouse and other organisms (19). In our case, it is especially true of the two parents of the BXD family (20). Here, we have systemically analyzed the potential causal role of Ace 2 expression in disease sensitivity and severity. Our focus is on networks of transcripts that covary with differences in Ace 2 expression after H1N1 infection. Expression of Ace 2 in lung is markedly but differentially decreased in a timedependent manner. Expression variation is negatively correlated with body weight loss and with differences in survival time after 


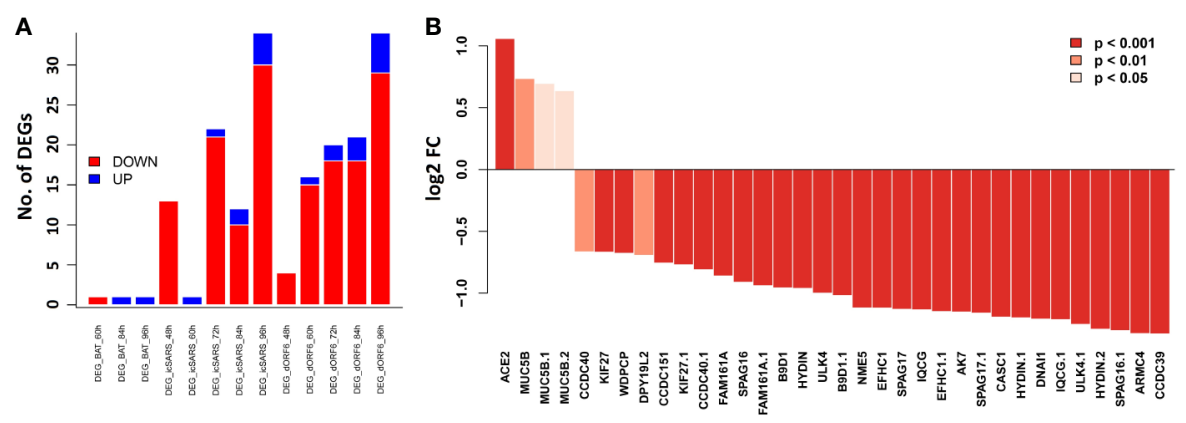

FIGURE 7 | Bar plots of the cilium function related genes in the SARS-CoV infected HAE cells. Primary human HAE cells were infected with MOI 3 SARS-CoV (icSARS-CoV), ic-SARS-CoV ORF6 and BAT-SARS_CoV and gene expression was analyzed by arrays. (A) Number of up-and downregulated differentially expressed genes for cilium function related genes that had orthologous genes in humans and were represented on the array (adj. $p<0.05$ and log2 FC > 0.58); $y$-axis: number of differentially expressed genes. (B) Log2 FC differences in expression values between HAE cells infected with icSARS-CoV at 96_h pi and mock-treated controls for cilium function related genes that had orthologous genes in humans and were represented on the array; $y$-axis: differences in log2 FC expression levels between groups. Adjusted p-values were taken from the Limma analysis of the entire transcriptome data.

A body weight loss at 2 dpi

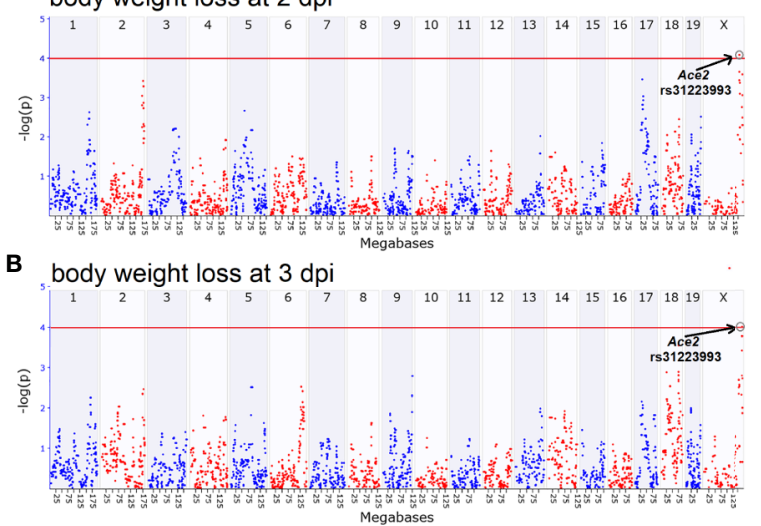

C

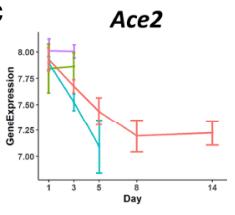

Maml3

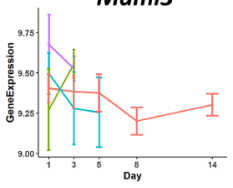

- B6 infected $\quad$ B6 mock

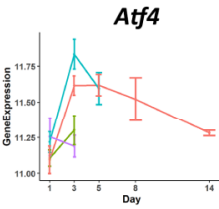

Muc5b
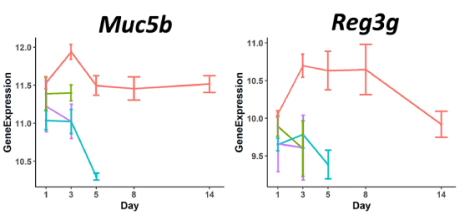

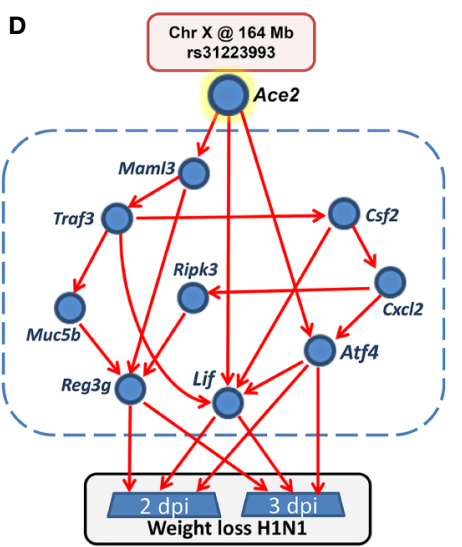

Cxcl2
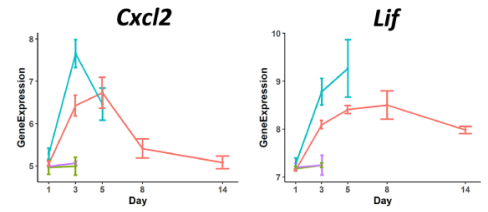

Ripk3
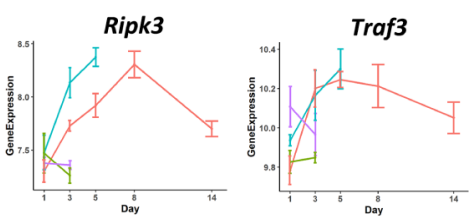

FIGURE 8 | A Bayesian network for the influenza disease severity in the BXD family. QTL maps of body weight loss at 2 (A) and 3 dpi (B) with peaks on Chr X at $164 \mathrm{Mb}$, respectively. The $x$-axis is chromosome position on a megabases (Mb) scale, while the $y$-axis gives the -log(p) of linkage. Each point on this plot is a single SNP marker used to evaluate linkage. The red line indicates the genome-wide significance threshold of $\sim 4$. (C) Kinetics of expression changes of the ten transcripts that are part of the Bayesian network. The $x$-axis defines days after infection, and the $y$-axis defines expression on a log2 scale. (D) Candidate Bayesian network comprising a top tier of genotypes on Chr X that predict weight loss (rs31223993), a middle tier within the blue dashed box of gene expression endophenotypes, and a bottom tier of body weight loss. 
infection. More importantly, the locus that modulates body weight loss at 2 and $3 \mathrm{dpi}$ is co-located precisely with the location of Ace 2 on distal Chr X. These results strongly suggest that Ace2 polymorphisms, or perhaps different isoforms as in humans (54), are linked to $\mathrm{H} 1 \mathrm{~N} 1$ disease progression, and that lower levels of Ace 2 may play a role in virus-induced ALI. This idea is corroborated by studies of $A c e 2^{-1-}$ knockouts infected with influenza H7N9 (17) or with respiratory syncytial virus (16). In both cases, lung pathology is more severe in the knockouts. These observations suggest that Ace 2 may mediate virus-induced ALI $(16,17)$. However, the underlying processes remain elusive and direct causes, versus reactive changes, are still not proved.

\section{Ace2 Correlated Genes Are Implicated in Inflammatory Related Pathways}

By using 41 isogenic RI strains, we defined Ace2-associated modules and molecular networks. We identified a total of $\sim 5,000$ Ace2correlated transcripts in infected lungs. As a set they are enriched in genes associated with respiratory and inflammatory networks and in several well understood pathways, including TNF, MAPK, and Notch signaling pathways. Extending this type of analysis to the corresponding proteomic, metabolomic, and metagenomic levels would provide a complementary and more accurate understanding of lung pathogenesis.

Key factors associated with $\mathrm{H} 1 \mathrm{~N} 1$-virus-induced lethality include virus-induced inflammatory cytokine storm and subsequent tissue destruction. Tumor necrosis factor (TNF) is a cytokine with multiple effects, including promoting cell growth, differentiation, apoptosis, and inducing inflammation $(57,58)$. TNF- $\alpha$, a member of the TNF family, can activate caspase protease, JNK, and transcription factor $\mathrm{NF}-\kappa \mathrm{B}$, thereby amplifying its roles in cytotoxicity, antiviral action, and immunoregulation. A previous study reported that inhibition of Tnf- $\alpha$ can protect mice from lethal $\mathrm{H} 1 \mathrm{~N} 1$ infection (59). In the present study, we found Ace 2 was negatively correlated $(r=-0.414, p=0.005)$ with the expression of $\operatorname{Tnf}$ and 34 other TNF signaling pathway genes, suggesting that Ace 2 may dampen excessive inflammatory immune responses by downregulating this signaling pathway and mitigating immune-mediated tissue destruction. We also highlighted 92 genes in the MAPK signaling pathway that covary with Ace2, including $T g f b 1$ ( $r=-0.459, p=0.002)$. Of note, it was shown that IAV can activate this pathway to support its replication (60). The authors proposed a model in which $T g f b 1$ acts as a pro-viral host factor by suppressing early immune responses, creating an environment conducive for IAV infection (61). Moreover, another set of 25 Ace2-correlated transcripts involved in the Notch signaling, most with negative covariation. This pathway is a bridge between antigen-presenting cells (APCs) and T cell communication circuits and plays a critical role in driving the immune system to overcome infectious disease (62).

\section{WGCNA Identifies Ace2 Co-Expressed Genes in the Respiratory Epithelium System}

There are essentially two parallel perturbation in this experiment-the actual infection with $\mathrm{H} 1 \mathrm{~N} 1$, and the natural genetic variation among these 43 diverse strains and their genomes. When performing WGCNA, a core of 461 transcripts covaried with Ace 2 after infection was identified (purple module). This set of covarying transcripts contained 31 genes that were related to ciliary function, including Ace 2 itself. Ace 2 expression may also modulate respiratory epithelial activity, although we stress that the causal polarity is still unknown.

Among the 31 prioritized cilium function related genes from the purple module (Table 1), Rnf128, Muc5b, Sox11, and Tff2 showed cis- or trans-regulation, and Tmprss2, Ccdc40, Ulk4, Cckar, Wdpcp, and Ift140 contained functional variants. Rnf128 encodes a ring-finger-containing protein which has been shown to possess E3 ubiquitin ligase activity (63). RNF128 is implicated in the induction of $\mathrm{T}$ cell anergic phenotype through inhibiting activation-induced IL2 and IL4 cytokine production (64) and mediating $\mathrm{T}$ cell receptor-CD3 degradation (65). In a recent study, Song et al. (66) reported that RNF128 promotes lysine-63linked ubiquitination and activation of a critical kinase TBK1, thereby augmenting the induction of type I interferon antiviral cytokines that curb viral replication. The authors' in vivo experiments further showed that $R n f 128$ deficient mice were more susceptible to vesicular stomatitis virus (an RNA virus) and herpes simplex virus (a DNA virus). MUC5B (mucin 5, subtype $B$, tracheobronchial), encodes a member of the mucin family of proteins, and is associated with idiopathic pulmonary fibrosis in humans (67). Overexpression of this gene in mice causes mucociliary dysfunction, and enhances lung fibrosis (68). It was reported to be required for airway defense in another study (69). TMPRSS2 (transmembrane protease, serine 2) encodes a protein that belongs to the serine protease family. Tmprss 2 knockout mice show reduced lung inflammation with IAVs (H1N1, H3N2, or H7N9) infection and exhibit decreased susceptibility to virusinduced morbidity/mortality (70). Studies also demonstrated that SARS coronavirus engages ACE2 as the entry receptor on the cell surface $(8,9)$ and employs the TMPRSS2 for Spike protein priming, which facilitates viral entry and infection $(71,72)$. CCDC40 (coiledcoil domain containing 40) encodes a protein that is essential for motile cilia function. According to the OMIM database, this gene is associated with recurrent respiratory infections (HP:0002205) and abnormal axonemal organization of respiratory motile cilia (HP:0012258). Malfunction of $C c d c 40$ in mice resulted in left pulmonary isomerism and lung situs inversus (73). In addition, Ulk4, Sox11, and Tff2 are associated with abnormal respiratory motile cilium (74), pulmonary hypoplasia (75), and abnormal adaptive immunity (76), respectively.

\section{Genetic Causal Pathways for the Host Responses to Infection}

The innate immune system plays a crucial role in the early recognition and subsequent triggering of proinflammatory responses. Our Bayesian network model suggests that this response may be modulated in part by TNF signaling pathway related genes (Atf4, Csf2, Cxcl2, lif, and Traf3), particularly Atf4 and Lif, two mediators for body weight loss at 2 and 3 dpi. Atf 4 encodes a transcription factor that was originally identified as a widely expressed mammalian DNA binding protein that could 
bind a tax-responsive enhancer element in the LTR of HTLV-1. Coronavirus replication is structurally and functionally associated with the endoplasmic reticulum (ER). The overexpression of the spike protein of SARS-CoV, MHV, and $\mathrm{HCoV}-\mathrm{HKU} 1 \mathrm{has}$ been shown to induce potent ER stress in cell culture (77-80). In response to ER stress, phosphorylated eIF2 $\alpha$ induces the expression of ATF4 (81). A previous study has demonstrated the protective effect of Ang 1-7 against Ang II induced ER stress and endothelial dysfunction (82). It indicates that the expression levels of Ace2 and Atf4 show a negative correlation, which is consistent with the results of our analysis. Furthermore, as an ER sensor, ATF4 defends lungs via induction of heme oxygenase 1 and its dependent responsiveness to ER stress is significantly impaired in the elderly (83). Lif (leukemia inhibitory factor) encodes a pleiotropic cytokine with roles in several different systems (http://www.informatics.jax.org/ marker/MGI:96787). A study has shown that LIF is expressed in the airspace of pneumonic lungs and the endogenous LIF facilitates tissue protection during pneumonia (84). In addition, The LIF-STAT3 axis has been identified as a key determinant of lung injury (84). The proactive role of Lif on lungs during infection was also demonstrated by another study (85). Therefore, Metcalfe proposed to use LIF to protect the lungs and reduce disease severity caused by SARS-CoV-2 (86).

Immune responses not only act to control and eliminate infections, but can inflict collateral damage of host lungs and other tissues. A diversity of molecules, metabolites, and cell types are involved including some of genes implicated in host defense against pathogens (Atf4, Cxcl2, Lif, Muc5b, Reg3g, Ripk3, and Traf3) in the Bayesian network. The key genetic driver of this damage in the BXD family is a locus on Chr X at $164 \mathrm{Mb}$, precisely the position of Ace2. This root causal path to body weight loss is mediated in part by variation in Ace 2 and Reg3g expression (regenerating islet-derived $3 \gamma$ ). The protein encoded by this gene is an antimicrobial lectin with activity against Grampositive bacteria. Reg3g is a critical STAT3 target in lung epithelium, and is also highly expressed in gastrointestinal epithelium (87). Our previous study revealed that Reg3g was significantly up-regulated in B6 but not in D2 after the H1N1 infection. Reg3g knockout mice on the B6 background showed a decrease in body weight at 4-6 dpi. (20). STAT3-mediated induction of the antimicrobial protein REG3G is required for host defense against methicillin-resistant Staphylococcus aureus pneumonia (87). A study has shown that IL22 and IL6 influence Reg3g expression in the gut and lungs, respectively (88). Gastrointestinal delivery of recombinant REG3G decreased lung inflammatory gene expression and protected $I l 22^{-/-}$mice from weight-loss during infection (88).

\section{Implications for COVID-19}

The ongoing outbreak of the COVID-19 caused by SARS-CoV-2 poses a global public health threat (89-91). SARS-CoV-2 entry into host cells is partly dependent on ACE2 (10), the receptor for SARS-CoV $(8,9)$. How ACE2 interacts with other proteins involved in the progression of SARS-CoV-2-induced respiratory diseases remains to be determined. The first report based on autopsy samples demonstrated that the pathological characteristics of COVID-19 are similar to those of SARS-CoV and MERS-CoV infected patients (92). We therefore focused most attention on 31 prioritized genes involved in cilium function (Table 1) that are co-expressed with Ace2 after H1N1 infection in mouse and in SARS-CoV-infected human HAE cells. Twenty out of these 31 transcripts are consistently up- or downregulated, suggesting these two viruses share common Ace2 networks that participate in the development and severity of lung pathology. In addition, our Bayesian network model also suggests several genes could be targeted for lung protection during SARS-CoV-2 infection, such as Atf4, Lif, and Reg3g.

\section{DATA AVAILABILITY STATEMENT}

The datasets presented in this study can be found in online repositories. The names of the repository/repositories and accession number(s) can be found at https://www.genenetwork. org/(GN807), https://www.ncbi.nlm.nih.gov/(GSE66040), and https://www.ncbi.nlm.nih.gov/(GSE47963).

\section{ETHICS STATEMENT}

The animal work described herein was approved by the Institutional Animal Care and Use Committee (IACUC) at the University of Tennessee Health Science Center (UTHSC).

\section{AUTHOR CONTRIBUTIONS}

LL, KS, RW, and RB conceived the study. SB and AS conducted the experiments. FX, KS, YC, and RW performed data analysis. LL, FX, KS, RW, and JG wrote the manuscript. FX, KS, and JG prepared the figures and tables. RW, KL, DA, and CJ edited the manuscript. All authors contributed to the article and approved the submitted version.

\section{FUNDING}

This work was supported by an intra-mural grant from the Helmholtz-Association (Program Infection and Immunity), a start-up grant from the University of Tennessee Health Science Center, a NIH/NIAID Research Grant U19-AI100625 to KS, and NIH/NIAID Research Grants U19-AI100625, U01 AI149644, and U19 AI 109761 to RB. GeneNetwork is supported by R01 GM123489 and the UT Center for Integrative and Translational Genomics. Provision of cells by Scott H. Randell was supported by grants from the Cystic Fibrosis Foundation (BOUCHE19R0 and NIH (DK065988).

\section{ACKNOWLEDGMENTS}

Primary human bronchial epithelial cells were provided by Scott H. Randell (Marsico Lung Institute, Tissue Procurement and Cell 
Culture Core, The University of North Carolina at Chapel Hill, USA). The cells were obtained under protocol 03-1396 approved by the University of North Carolina at Chapel Hill Biomedical Institutional Review Board. The RNA-seq was carried out by the Molecular Resource Center at UTHSC.

\section{REFERENCES}

1. Jester BJ, Uyeki TM, Patel A, Koonin L, Jernigan DB. 100 years of medical countermeasures and pandemic influenza preparedness. Am J Public Health (2018) 108(11):1469-72. doi: 10.2105/AJPH.2018.304586

2. Hendrickson CM, Matthay MA. Viral pathogens and acute lung injury: investigations inspired by the SARS epidemic and the 2009 H1N1 influenza pandemic. Semin Respir Crit Care Med (2013) 34(4):475-86.

3. El Zowalaty ME, Järhult JD. From SARS to COVID-19: A previously unknown SARS-related coronavirus (SARS-CoV-2) of pandemic potential infecting humans-Call for a One Health approach. One Health (2020) 9:100124. doi: 10.1016/j.onehlt.2020.100124

4. Wang C, Pan R, Wan X, Tan Y, Xu L, Ho CS, et al. Immediate psychological responses and associated factors during the initial stage of the 2019 coronavirus disease (COVID-19) epidemic among the general population in china. Int J Environ Res Public Health (2020) 17(5):1729. doi: 10.3390/ ijerph 17051729

5. Team NS-OIAVI. Emergence of a novel swine-origin influenza A (H1N1) virus in humans. New Engl J Med (2009) 360(25):2605-15. doi: 10.1056/ NEJMoa0903810

6. Horby P, Nguyen NY, Dunstan SJ, Baillie JK. The role of host genetics in susceptibility to influenza: a systematic review. PloS One (2012) 7(3):e33180. doi: 10.1371/journal.pone.0033180

7. Horby P, Sudoyo H, Viprakasit V, Fox A, Thai P, Yu H, et al. What is the evidence of a role for host genetics in susceptibility to influenza $\mathrm{A} / \mathrm{H} 5 \mathrm{~N} 1$ ? Epidemiol Infect (2010) 138(11):1550-8. doi: 10.1017/S0950268810000518

8. Kuba K, Imai Y, Rao S, Gao H, Guo F, Guan B, et al. A crucial role of angiotensin converting enzyme 2 (ACE2) in SARS coronavirus-induced lung injury. Nat Med (2005) 11(8):875-9. doi: 10.1038/nm1267

9. Li W, Moore MJ, Vasilieva N, Sui J, Wong SK, Berne MA, et al. Angiotensinconverting enzyme 2 is a functional receptor for the SARS coronavirus. Nature (2003) 426(6965):450-4. doi: 10.1038/nature02145

10. Zhang H, Penninger JM, Li Y, Zhong N, Slutsky AS. Angiotensin-converting enzyme 2 (ACE2) as a SARS-CoV-2 receptor: molecular mechanisms and potential therapeutic target. Intensive Care Med (2020) 46(4):586-90. doi: 10.1007/s00134-020-05985-9

11. Zhao Y, Zhao Z, Wang Y, Zhou Y, Ma Y, Zuo W. Single-cell RNA expression profiling of ACE2, the putative receptor of Wuhan 2019-nCov. BioRxiv (2020). doi: 10.1101/2020.01.26.919985

12. Sims AC, Burkett SE, Yount B, Pickles RJ. SARS-CoV replication and pathogenesis in an in vitro model of the human conducting airway epithelium. Virus Res (2008) 133(1):33-44. doi: 10.1016/j.virusres.2007.03.013

13. Kuba K, Imai Y, Penninger JM. Angiotensin-converting enzyme 2 in lung diseases. Curr Opin Pharmacol (2006) 6(3):271-6. doi: 10.1016/j.coph. 2006.03.001

14. Imai Y, Kuba K, Rao S, Huan Y, Guo F, Guan B, et al. Angiotensin-converting enzyme 2 protects from severe acute lung failure. Nature (2005) 436 (7047):112-6. doi: 10.1038/nature03712

15. Liu X, Yang N, Tang J, Liu S, Luo D, Duan Q, et al. Downregulation of angiotensin-converting enzyme 2 by the neuraminidase protein of influenza $\mathrm{A}$ (H1N1) virus. Virus Res (2014) 185:64-71. doi: 10.1016/j.virusres.2014.03.010

16. Gu H, Xie Z, Li T, Zhang S, Lai C, Zhu P, et al. Angiotensin-converting enzyme 2 inhibits lung injury induced by respiratory syncytial virus. Sci Rep (2016) 6:19840. doi: 10.1038/srep19840

17. Yang $\mathrm{P}, \mathrm{Gu} \mathrm{H}$, Zhao Z, Wang W, Cao B, Lai C, et al. Angiotensin-converting enzyme 2 (ACE2) mediates influenza $\mathrm{H} 7 \mathrm{~N} 9$ virus-induced acute lung injury. Sci Rep (2014) 4:7027. doi: 10.1038/srep07027

18. Consortium G. The Genotype-Tissue Expression (GTEx) pilot analysis: multitissue gene regulation in humans. Science (2015) 348(6235):648-60. doi: $10.1126 /$ science. 1262110

\section{SUPPLEMENTARY MATERIAL}

The Supplementary Material for this article can be found online at: https://www.frontiersin.org/articles/10.3389/fimmu.2020. 607314/full\#supplementary-material

19. Srivastava B, Błażejewska P, Heßmann M, Bruder D, Geffers R, Mauel S, et al. Host genetic background strongly influences the response to influenza a virus infections. PloS One (2009) 4(3):e4857. doi: 10.1371/journal.pone.0004857

20. Wilk E, Pandey AK, Leist SR, Hatesuer B, Preusse M, Pommerenke C, et al. RNAseq expression analysis of resistant and susceptible mice after influenza A virus infection identifies novel genes associated with virus replication and important for host resistance to infection. BMC Genomics (2015) 16(1):655. doi: 10.1186/s12864-015-1867-8

21. Williams RW, Gu J, Qi S, Lu L. The genetic structure of recombinant inbred mice: high-resolution consensus maps for complex trait analysis. Genome Biol (2001) 2(11):research0046. 1. doi: 10.1186/gb-2001-2-11-research0046

22. Ashbrook DG, Arends D, Prins P, Mulligan MK, Roy S, Williams EG, et al. The expanded BXD family of mice: A cohort for experimental systems genetics and precision medicine. bioRxiv (2019) 672097. doi: 10.1101/672097

23. Nedelko T, Kollmus H, Klawonn F, Spijker S, Lu L, Heßman M, et al. Distinct gene loci control the host response to influenza H1N1 virus infection in a time-dependent manner. BMC Genomics (2012) 13(1):411. doi: 10.1186/14712164-13-411

24. Miyairi I, Tatireddigari VRA, Mahdi OS, Rose LA, Belland RJ, Lu L, et al. The p47 GTPases Iigp2 and Irgb10 regulate innate immunity and inflammation to murine Chlamydia psittaci infection. J Immunol (2007) 179(3):1814-24. doi: 10.4049/jimmunol.179.3.1814

25. Miyairi I, Laxton JD, Wang X, Obert CA, Arva Tatireddigari VR, van Rooijen N, et al. Chlamydia psittaci genetic variants differ in virulence by modulation of host immunity. J Infect Dis (2011) 204(4):654-63. doi: 10.1093/infdis/jir333

26. Miyairi I, Ziebarth J, Laxton JD, Wang X, Van Rooijen N, Williams RW, et al. Host genetics and Chlamydia disease: prediction and validation of disease severity mechanisms. PloS One (2012) 7(3):e33781. doi: 10.1371/journal.pone.0033781

27. Boon AC, DeBeauchamp J, Hollmann A, Luke J, Kotb M, Rowe S, et al. Host genetic variation affects resistance to infection with a highly pathogenic H5N1 influenza A virus in mice. J Virol (2009) 83(20):10417-26. doi: 10.1128/ JVI.00514-09

28. Blazejewska P, Koscinski L, Viegas N, Anhlan D, Ludwig S, Schughart K. Pathogenicity of different PR8 influenza A virus variants in mice is determined by both viral and host factors. Virology (2011) 412(1):36-45. doi: 10.1016/j.virol.2010.12.047

29. Pommerenke C, Wilk E, Srivastava B, Schulze A, Novoselova N, Geffers R et al. Global transcriptome analysis in influenza-infected mouse lungs reveals the kinetics of innate and adaptive host immune responses. PloS One (2012) 7 (7):e41169. doi: 10.1371/journal.pone.0041169

30. Alberts R, Srivastava B, Wu H, Viegas N, Geffers R, Klawonn F, et al. Gene expression changes in the host response between resistant and susceptible inbred mouse strains after influenza A infection. Microbes Infect (2010) 12 (4):309-18. doi: 10.1016/j.micinf.2010.01.008

31. Krueger F. Trim galore. A wrapper tool around Cutadapt and FastQC to consistently apply quality and adapter trimming to FastQ files. Available at: http://www.bioinformatics.babraham.ac.uk/projects/trim_galore/

32. Dobin A, Davis CA, Schlesinger F, Drenkow J, Zaleski C, Jha S, et al. STAR: ultrafast universal RNA-seq aligner. Bioinformatics (2013) 29(1):15-21. doi: 10.1093/bioinformatics/bts635

33. Liao Y, Smyth GK, Shi W. The R package Rsubread is easier, faster, cheaper and better for alignment and quantification of RNA sequencing reads. Nucleic Acids Res (2019) 47(8):e47-e. doi: 10.1093/nar/gkz114

34. Love MI, Huber W, Anders S. Moderated estimation of fold change and dispersion for RNA-seq data with DESeq2. Genome Biol (2014) 15(12):550. doi: 10.1186/s13059-014-0550-8

35. Leek JT, Johnson WE, Parker HS, Jaffe AE, Storey JD. The sva package for removing batch effects and other unwanted variation in high-throughput experiments. Bioinformatics (2012) 28(6):882-3. doi: 10.1093/bioinformatics/ bts034 
36. Johnson WE, Li C, Rabinovic A. Adjusting batch effects in microarray expression data using empirical Bayes methods. Biostatistics (2007) 8 (1):118-27. doi: 10.1093/biostatistics/kxj037

37. Durinck S, Spellman PT, Birney E, Huber W. Mapping identifiers for the integration of genomic datasets with the R/Bioconductor package biomaRt. Nat Protoc (2009) 4(8):1184. doi: 10.1038/nprot.2009.97

38. Zhou X, Stephens M. Genome-wide efficient mixed-model analysis for association studies. Nat Genet (2012) 44(7):821-4. doi: 10.1038/ng.2310

39. Team RC. R: A language and environment for statistical computing. Vienna, Austria: R Foundation for Statistical Computing (2013).

40. Langfelder P, Horvath S. WGCNA: an R package for weighted correlation network analysis. BMC Bioinf (2008) 9(1):559. doi: 10.1186/1471-2105-9-559

41. Liao Y, Wang J, Jaehnig EJ, Shi Z, Zhang B. WebGestalt 2019: gene set analysis toolkit with revamped UIs and APIs. Nucleic Acids Res (2019) 47(W1):W199205. doi: 10.1093/nar/gkz401

42. Mulligan MK, Mozhui K, Prins P, Williams RW. GeneNetwork: a toolbox for systems genetics. Systems Genetics. New York, NY: Humana Press (2017). p. 75-120. doi: 10.1007/978-1-4939-6427-7_4

43. Chesler EJ, Lu L, Shou S, Qu Y, Gu J, Wang J, et al. Complex trait analysis of gene expression uncovers polygenic and pleiotropic networks that modulate nervous system function. Nat Genet (2005) 37(3):233. doi: 10.1038/ng1518

44. Feenstra B, Skovgaard IM, Broman KW. Mapping quantitative trait loci by an extension of the Haley-Knott regression method using estimating equations. Genetics (2006) 173(4):2269-82. doi: 10.1534/genetics.106.058537

45. Wang X, Pandey AK, Mulligan MK, Williams EG, Mozhui K, Li Z, et al. Joint mouse-human phenome-wide association to test gene function and disease risk. Nat Commun (2016) 7(1):1-13. doi: 10.1038/ncomms10464

46. Ziebarth JD, Bhattacharya A, Cui Y. Bayesian Network Webserver: a comprehensive tool for biological network modeling. Bioinformatics (2013) 29(21):2801-3. doi: 10.1093/bioinformatics/btt472

47. Ziebarth JD, Cui Y. Multiscalar genetic pathway modeling with hybrid Bayesian networks. Wiley Interdiscip Rev: Comput Stat (2019) 0(0):e1479. doi: 10.1002/wics.1479

48. Ziebarth JD, Cui Y. Precise network modeling of systems genetics data using the Bayesian network webserver. Methods Mol Biol (2017) 319-35. doi: 10.1007/978-1-4939-6427-7_15

49. Benjamini Y, Hochberg Y. Controlling the false discovery rate: a practical and powerful approach to multiple testing. J R Stat Soc: Ser B (Methodological) (1995) 57(1):289-300. doi: 10.1111/j.2517-6161.1995.tb02031.x

50. Sims AC, Tilton SC, Menachery VD, Gralinski LE, Schäfer A, Matzke MM, et al. Release of severe acute respiratory syndrome coronavirus nuclear import block enhances host transcription in human lung cells. J Virol (2013) 87 (7):3885-902. doi: 10.1128/JVI.02520-12

51. Becker MM, Graham RL, Donaldson EF, Rockx B, Sims AC, Sheahan T, et al. Synthetic recombinant bat SARS-like coronavirus is infectious in cultured cells and in mice. Proc Natl Acad Sci (2008) 105(50):19944-9. doi: 10.1073/ pnas.0808116105

52. Smyth Gordon K. Linear models and empirical bayes methods for assessing differential expression in microarray experiments. Stat Appl Genet Mol Biol (2004) 3(1):1-25. doi: 10.2202/1544-6115.1027

53. Sims AC, Baric RS, Yount B, Burkett SE, Collins PL, Pickles RJ. Severe acute respiratory syndrome coronavirus infection of human ciliated airway epithelia: role of ciliated cells in viral spread in the conducting airways of the lungs. J Virol (2005) 79(24):15511-24. doi: 10.1128/JVI.79.24.15511-15524.2005

54. Onabajo OO, Banday AR, Stanifer ML, Yan W, Obajemu A, Santer DM, et al. Interferons and viruses induce a novel truncated ACE2 isoform and not the full-length SARS-CoV-2 receptor. Nat Genet (2020) 52:1283-93. doi: 10.1101/ 2020.07.19.210955

55. Ng KW, Attig J, Bolland W, Young GR, Major J, Wrobel AG, et al. Tissuespecific and interferon-inducible expression of nonfunctional ACE2 through endogenous retroelement co-option. Nat Genet (2020) 52:1294-302. doi: 10.1101/2020.07.24.219139

56. Trammell RA, Toth LA. Genetic susceptibility and resistance to influenza infection and disease in humans and mice. Expert Rev Mol Diagn (2008) 8 (4):515-29. doi: 10.1586/14737159.8.4.515

57. Holbrook J, Lara-Reyna S, Jarosz-Griffiths H, McDermott M. Tumour necrosis factor signalling in health and disease. F1000Research (2019) 8:111-23. doi: 10.12688/f1000research.17023.1
58. Sedger LM, McDermott MF. TNF and TNF-receptors: From mediators of cell death and inflammation to therapeutic giants-past, present and future. Cytokine Growth factor Rev (2014) 25(4):453-72. doi: 10.1016/j.cytogfr.2014.07.016

59. Shi X, Zhou W, Huang H, Zhu H, Zhou P, Zhu H, et al. Inhibition of the inflammatory cytokine tumor necrosis factor-alpha with etanercept provides protection against lethal H1N1 influenza infection in mice. Crit Care (2013) 17(6):R301. doi: 10.1186/cc13171

60. Wang X, Tan J, Zoueva O, Zhao J, Ye Z, Hewlett I. Novel pandemic influenza A (H1N1) virus infection modulates apoptotic pathways that impact its replication in A549 cells. Microbes Infect (2014) 16(3):178-86. doi: 10.1016/ j.micinf.2013.11.003

61. Denney L, Branchett W, Gregory LG, Oliver RA, Lloyd CM. Epithelial-derived TGF- $\beta 1$ acts as a pro-viral factor in the lung during influenza A infection. Mucosal Immunol (2018) 11(2):523-35. doi: 10.1038/mi.2017.77

62. Ito T, Allen RM, Carson IV WF, Schaller M, Cavassani KA, Hogaboam CM, et al. The critical role of Notch ligand Delta-like 1 in the pathogenesis of influenza A virus (H1N1) infection. PloS Pathog (2011) 7(11):e1002341. doi: 10.1371/journal.ppat.1002341

63. Zhang Q, Meng Y, Zhang L, Chen J, Zhu D. RNF13: a novel RING-type ubiquitin ligase over-expressed in pancreatic cancer. Cell Res (2009) 19 (3):348-57. doi: 10.1038/cr.2008.285

64. Anandasabapathy N, Ford GS, Bloom D, Holness C, Paragas V, Seroogy C, et al. GRAIL: an E3 ubiquitin ligase that inhibits cytokine gene transcription is expressed in anergic CD4+ T cells. Immunity (2003) 18(4):535-47. doi: 10.1016/S1074-7613(03)00084-0

65. Nurieva RI, Zheng S, Jin W, Chung Y, Zhang Y, Martinez GJ, et al. The E3 ubiquitin ligase GRAIL regulates $\mathrm{T}$ cell tolerance and regulatory $\mathrm{T}$ cell function by mediating $\mathrm{T}$ cell receptor-CD3 degradation. Immunity (2010) 32(5):670-80. doi: 10.1016/j.immuni.2010.05.002

66. Song G, Liu B, Li Z, Wu H, Wang P, Zhao K, et al. E3 ubiquitin ligase RNF128 promotes innate antiviral immunity through K63-linked ubiquitination of TBK1. Nat Immunol (2016) 17(12):1342. doi: 10.1038/ni.3588

67. Peljto AL, Zhang Y, Fingerlin TE, Ma S-F, Garcia JG, Richards TJ, et al. Association between the MUC5B promoter polymorphism and survival in patients with idiopathic pulmonary fibrosis. Jama (2013) 309(21):2232-9. doi: 10.1001/jama.2013.5827

68. Hancock LA, Hennessy CE, Solomon GM, Dobrinskikh E, Estrella A, Hara N, et al. Muc5b overexpression causes mucociliary dysfunction and enhances lung fibrosis in mice. Nat Commun (2018) 9(1):1-10. doi: 10.1038/s41467-018-07768-9

69. Roy MG, Livraghi-Butrico A, Fletcher AA, McElwee MM, Evans SE, Boerner RM, et al. Muc5b is required for airway defence. Nature (2014) 505 (7483):412-6. doi: 10.1038/nature12807

70. Sakai K, Ami Y, Tahara M, Kubota T, Anraku M, Abe M, et al. The host protease TMPRSS2 plays a major role in in vivo replication of emerging H7N9 and seasonal influenza viruses. J Virol (2014) 88(10):5608-16. doi: 10.1128/JVI.03677-13

71. Glowacka I, Bertram S, Müller MA, Allen P, Soilleux E, Pfefferle S, et al. Evidence that TMPRSS2 activates the severe acute respiratory syndrome coronavirus spike protein for membrane fusion and reduces viral control by the humoral immune response. J Virol (2011) 85(9):4122-34. doi: 10.1128/JVI.02232-10

72. Hoffmann M, Kleine-Weber H, Schroeder S, Krüger N, Herrler T, Erichsen S, et al. SARS-CoV-2 cell entry depends on ACE2 and TMPRSS2 and is blocked by a clinically proven protease inhibitor. Cell (2020) 181(2):271-80. doi: 10.1016/j.cell.2020.02.052

73. Becker-Heck A, Zohn IE, Okabe N, Pollock A, Lenhart KB, Sullivan-Brown J, et al. The coiled-coil domain containing protein CCDC40 is essential for motile cilia function and left-right axis formation. Nat Genet (2011) 43(1):79. doi: 10.1038/ng.727

74. Vogel P, Read R, Hansen G, Payne B, Small D, Sands A, et al. Congenital hydrocephalus in genetically engineered mice. Vet Pathol (2012) 49(1):16681. doi: $10.1177 / 0300985811415708$

75. Sock E, Rettig SD, Enderich J, Bösl MR, Tamm ER, Wegner M. Gene targeting reveals a widespread role for the high-mobility-group transcription factor Sox11 in tissue remodeling. Mol Cell Biol (2004) 24(15):6635-44. doi: 10.1128/ MCB.24.15.6635-6644.2004

76. Baus-Loncar M, Schmid J, Lalani E-N, Rosewell I, Goodlad R, Stamp G, et al. Trefoil factor 2 (TFF2) deficiency in murine digestive tract influences the immune system. Cell Physiol Biochem (2005) 16(1-3):31-42. doi: 10.1159/ 000087729 
77. Fung TS, Huang M, Liu DX. Coronavirus-induced ER stress response and its involvement in regulation of coronavirus-host interactions. Virus Res (2014) 194:110-23. doi: 10.1016/j.virusres.2014.09.016

78. Chan C-P, Siu K-L, Chin K-T, Yuen K-Y, Zheng B, Jin D-Y. Modulation of the unfolded protein response by the severe acute respiratory syndrome coronavirus spike protein. J Virol (2006) 80(18):9279-87. doi: 10.1128/JVI.00659-06

79. Versteeg GA, Van De Nes PS, Bredenbeek PJ, Spaan WJ. The coronavirus spike protein induces endoplasmic reticulum stress and upregulation of intracellular chemokine mRNA concentrations. J Virol (2007) 81 (20):10981-90. doi: 10.1128/JVI.01033-07

80. Siu K-L, Chan C-P, Kok K-H, Woo PC, Jin D-Y. Comparative analysis of the activation of unfolded protein response by spike proteins of severe acute respiratory syndrome coronavirus and human coronavirus HKU1. Cell Biosci (2014) 4(1):1-9. doi: 10.1186/2045-3701-4-3

81. Nguyen H, Uhal BD. The unfolded protein response controls ER stressinduced apoptosis of lung epithelial cells through angiotensin generation. Am J Physiology-Lung Cell Mol Physiol (2016) 311(5):L846-54. doi: 10.1152/ ajplung.00449.2015

82. Murugan D, Lau YS, Lau WC, Mustafa MR, Huang Y. Angiotensin 1-7 protects against angiotensin II-induced endoplasmic reticulum stress and endothelial dysfunction via mas receptor. PloS One (2015) 10(12):e0145413. doi: 10.1371/journal.pone.0145413

83. Abouhashem AS, Singh K, Azzazy HM, Sen CK. Is Low Alveolar Type II Cell SOD3 in the Lungs of Elderly Linked to the Observed Severity of COVID-19? Antioxid Redox Signaling (2020) 33(2):59-65. doi: 10.1089/ars.2020.8111

84. Quinton LJ, Mizgerd JP, Hilliard KL, Jones MR, Kwon CY, Allen E. Leukemia inhibitory factor signaling is required for lung protection during pneumonia. J Immunol (2012) 188(12):6300-8. doi: 10.4049/jimmunol.1200256

85. Foronjy RF, Dabo AJ, Cummins N, Geraghty P. Leukemia inhibitory factor protects the lung during respiratory syncytial viral infection. BMC Immunol (2014) 15(1):41. doi: 10.1186/s12865-014-0041-4

86. Metcalfe SM. COVID-19 lockdown: de-risking exit by protecting the lung with leukaemia inhibitory factor (LIF). Med Drug Discovery (2020) 6:100043. doi: 10.1016/j.medidd.2020.100043
87. Choi S-M, McAleer JP, Zheng M, Pociask DA, Kaplan MH, Qin S, et al. Innate Stat3-mediated induction of the antimicrobial protein Reg3 $\gamma$ is required for host defense against MRSA pneumonia. J Exp Med (2013) 210(3):551-61. doi: 10.1084/jem.20120260

88. McAleer JP, Nguyen NL, Chen K, Kumar P, Ricks DM, Binnie M, et al. Pulmonary Th17 antifungal immunity is regulated by the gut microbiome. J Immunol (2016) 197(1):97-107. doi: 10.4049/jimmunol. 1502566

89. Lai C-C, Shih T-P, Ko W-C, Tang H-J, Hsueh P-R. Severe acute respiratory syndrome coronavirus 2 (SARS-CoV-2) and corona virus disease-2019 (COVID-19): the epidemic and the challenges. Int $J$ Antimicrob Agents (2020) 55(3):105924. doi: 10.1016/j.ijantimicag.2020.105924

90. Ahmad T, Rodriguez-Morales AJ. Emergence of COVID-19 (formerly 2019 novel Coronavirus): a new threat from China. Rev Panamericana Enfermedades Infecciosas (2020) 2(2):37-8. doi: 10.13140/RG.2.2.25239.93607

91. Yee J, Unger L, Zadravecz F, Cariello P, Seibert A, Johnson MA, et al. Novel coronavirus 2019 (COVID-19): Emergence and implications for emergency care. J Am Coll Emergency Physicians Open (2020) 1(2):63-9. doi: 10.1002/ emp2.12034

92. Xu Z, Shi L, Wang Y, Zhang J, Huang L, Zhang C, et al. Pathological findings of COVID-19 associated with acute respiratory distress syndrome. Lancet Respir Med (2020) 8(4):450-2. doi: 10.1016/S2213-2600(20)30076-X

Conflict of Interest: The authors declare that the research was conducted in the absence of any commercial or financial relationships that could be construed as a potential conflict of interest.

Copyright (c) 2021 Xu, Gao, Bergmann, Sims, Ashbrook, Baric, Cui, Jonsson, Li, Williams, Schughart and Lu. This is an open-access article distributed under the terms of the Creative Commons Attribution License (CC BY). The use, distribution or reproduction in other forums is permitted, provided the original author(s) and the copyright owner(s) are credited and that the original publication in this journal is cited, in accordance with accepted academic practice. No use, distribution or reproduction is permitted which does not comply with these terms. 